\title{
Monitoring and Managing Cardiovascular Risk in Immune Mediated Inflammatory Diseases
}

\author{
Panagiota Anyfanti \\ Athanasia Dara ${ }^{2}$ \\ Elena Angeloudi ${ }^{1}$ \\ Eleni Bekiari (D) \\ Theodoros Dimitroulas ${ }^{2}$ \\ George D Kitas ${ }^{3,4}$
}

'Second Medical Department, Hippokration Hospital, Aristotle University of Thessaloniki, Thessaloniki, Greece; ${ }^{2}$ Fourth Department of Internal Medicine, Hippokration Hospital, Aristotle University of Thessaloniki, Thessaloniki, Greece; ${ }^{3}$ Department of Rheumatology, Russells Hall Hospital, Dudley Group NHS Foundation Trust, Dudley, UK; ${ }^{4}$ School of Sport, Exercise and Rehabilitation Sciences, University of Birmingham, Birmingham, UK
Correspondence: Panagiota Anyfanti Hippokration General Hospital, 49, Konstantinoupoleos str, 54642,

Thessaloniki, Greece

Tel +302310 892244

Fax +302310512378

Email panyfan@hotmail.com

George D Kitas

Dudley Group NHS Foundation Trust,

Russells Hall Hospital, Clinical Research

Unit, Dudley, DYI 2HQ, UK

Email george.kitas@nhs.net

\begin{abstract}
Cardiovascular disease (CVD) is common in immune-mediated inflammatory diseases (IMIDs) and it is predominately attributed to the interplay between chronic inflammation and traditional CVD risk factors. CVD has significant impact on the survival of patients with IMIDs as it is associated with increased morbidity and mortality. Despite recommendations for monitoring and managing CVD in patients with IMIDs, the individual CVD risk assessment remains problematic as CVD risk calculators for the general population consistently underestimate the risk in patients with IMIDs. Application of new technologies utilizing artificial intelligence techniques have shown promising potential for tailoring predictive medicine to the individual patient, but further validation of their role in clinical decision-making is warranted. In the meantime, individuals with IMIDs should be encouraged to adopt behavioral interventions targeting at modifiable lifestyle CVD risk factors, whereas rheumatologists need to be well aware of the unfavorable effects of antirheumatic medication on various CVD risk factors and outcomes. In the current paper, we aim to provide an overview of current and emerging strategies for mitigating CVD risk in patients with IMIDs, based on a practical approach.
\end{abstract}

Keywords: chronic inflammatory diseases, cardiovascular risk assessment, lifestyle modifications, pharmacological management

\section{Introduction}

Immune-mediated inflammatory diseases (IMIDs) are associated with reduced life expectancy compared to the general population despite the implementation of targeted management strategies (eg treat- to-target) and the availability of novel therapies such as biologic and/or targeted disease modifying antirheumatic drugs (DMARDs). ${ }^{1,2}$ The major part of the excess mortality is attributed to comorbidities, predominantly cardiovascular disease (CVD) and lung involvement. ${ }^{3-6}$

Particularly in view of CVD, clinical phenotype can vary greatly between different inflammatory conditions ranging from macrovascular atherosclerotic disease to non-ischaemic heart failure, coronary microvascular dysfunction, cardiac autonomic neuropathy and arrhythmias, depending on disease-specific characteristics. ${ }^{7}$ For example, the high-grade chronic inflammatory burden in rheumatoid arthritis (RA), the adverse metabolic profile in psoriatic arthritis (PsA), the presence of anti-endothelial cell and anti-phospholipid antibodies in systemic lupus erythematosus (SLE) as well as the fibrotic process in systemic sclerosis represent amongst others - distinct pathogenic mechanisms promoting CVD comorbidities in different IMIDs. ${ }^{8,9}$ However, atherosclerosis appears to pose the greatest CVD risk across the whole spectrum of IMIDs, as systemic inflammation and autoimmune 
activation - the main players in immunopathology of IMIDs - are actively involved in all stages of vascular injury, from endothelial dysfunction to plaque formation and rupture. ${ }^{10,11}$ In addition, cumulative inflammation provides a set of pathways that link traditional CVD risk factors such as hypertension, dyslipidaemia, obesity, insulin resistance and physical inactivity, all of which are highly prevalent in IMIDs, ${ }^{12-15}$ with vascular alterations that promote atherosclerosis and its complications. ${ }^{16}$

The awareness of increased risk has led to evidencebased practice recommendations for managing CVD risk in IMIDs. ${ }^{17}$ The main domains of such preventive strategies include effective control of systemic inflammation, management of classic CVD risk factors and lifestyle changes, ${ }^{18}$ taking also into account that a number of antirheumatic drugs may affect CVD risk positively or negatively. ${ }^{19,20}$ The aim of the current paper is to provide an overview of current and emerging strategies for mitigating CVD risk in IMIDs, with a special focus on assessment of comprehensive individual risk via risk algorithms and relevant recommendations, non-pharmacological interventions and disease modifying drugs (DMARDs) modifications according to cardiovascular comorbidities and disease-related risk profile of the patients. Aspects of applications of new technologies in the assessment and management of cardiovascular comorbidities will also be discussed.

\section{Risk Factors of CVD Disease in IMIDs}

The increased CVD risk associated with IMIDs is well acknowledged and has been extensively described in RA, SLE, and, more recently, PsA and ankylosing spondylitis. ${ }^{21}$ Several efforts have been made to unveil the underlying mechanisms of increased CVD risk in chronic inflammatory diseases, especially in RA, which are briefly presented in Figure 1. In particular, epidemiological data have consistently demonstrated increased prevalence of several traditional CVD risk factors, such as smoking, hypertension, dyslipidaemia, insulin resistance, obesity and impaired metabolic profile, physical and lack of exercise. ${ }^{8}$ However, their presence can only partially explain the excess CVD risk. Chronic systemic inflammation, triggered by dysregulation of autoimmune mechanisms, plays a pivotal role and appears to drastically interact with traditional risk factors in a bidirectional manner. $^{22,23}$ Notably, patients with inflammatory diseases

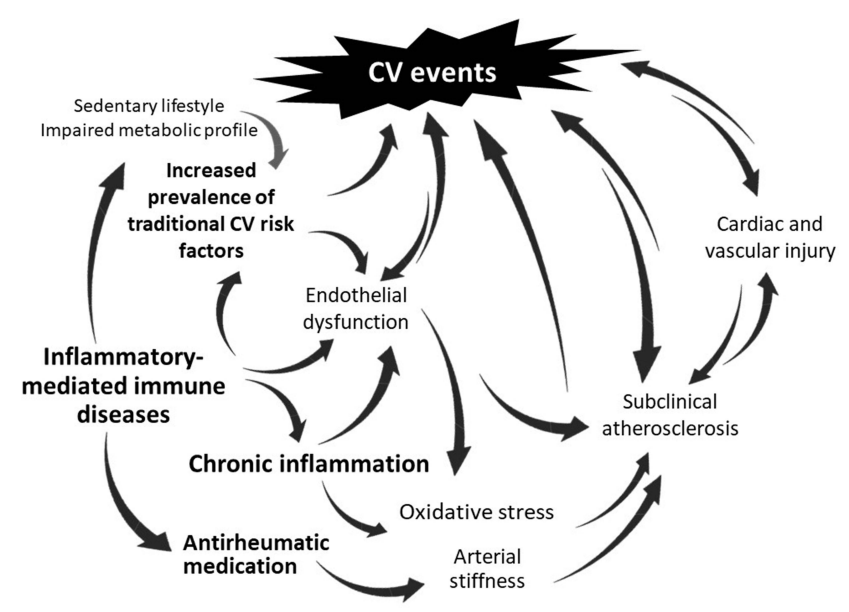

Figure I Factors implicated in the pathogenesis of increased cardiovascular disease (CVD) risk in patients with immune-mediated inflammatory diseases. Traditional CVD risk factors, disease-related factors triggered by the dysregulation of innate immunity, and the current and cumulative inflammatory burden, act synergistically and promote the pathophysiological processes of endothelial dysfunction, arterial stiffness and oxidative stress. The mutual interplay of these factors eventually leads to accelerated atherosclerosis, subclinical micro- and macrovascular damage and subsequently, clinically evident CVD.

such as RA present increased levels of circulating biomarkers of cardiac injury and endothelial dysfunction, which correlate with current and cumulative inflammatory load, ${ }^{24-26}$ as well as increased prevalence of subclinical vascular injury in divergent vascular beds, even in the absence of CVD comorbidities. ${ }^{27-29}$ The association between inflammation and atherosclerosis, the cornerstone of CVD, has been the subject of extensive investigation in recent years and the contribution of inflammation in the pathogenesis and progression of atherogenesis has been well demonstrated. ${ }^{23}$ Endothelial dysfunction, oxidative stress in vascular endothelial cells and macrophage accumulation, toll-like receptor signaling, pro-inflammatory cytokine production, such as tumor necrosis factor-alpha (TNF- $\alpha$ ), interleukin-1 (IL-1), IL-6, are only a few of the underlying mechanisms involved in the atherogenic process. ${ }^{30}$ Vice versa, adequate control of inflammation, either with conventional or biologic DMARDs, particularly anti-TNF- $\alpha$ agents and methotrexate, decelerates the atherogenic process. ${ }^{8}$ Taken together, the presence of traditional CVD risk factors along with high grade systemic inflammation appear to mutually determine the CVD risk of patients with IMIDs.

\section{Assessment of CVD Risk in IMIDs}

Although musculoskeletal and overall outcomes have been improving over the last decades in patients with IMIDs, the risk of fatal and nonfatal CVD events remains higher 
compared to general population. ${ }^{31}$ Amongst others, the inability to efficiently perform composite CVD risk assessment in patients with IMIDs using traditional populationbased risk tools may have significant input in these observations. The most widely used in the general population cardiovascular risk calculators are the Systematic Coronary Risk Evaluation (SCORE) calculator, ${ }^{32}$ and the Framingham Risk Score ${ }^{33}$ which has been previously replaced by the American College of Cardiology and American Heart Association (ACC/AHA) Pooled Cohort Equation risk calculator in the $2013 \mathrm{ACC} / \mathrm{AHA}$ guidelines on CVD prevention. ${ }^{34}$ These scores incorporate information on age, sex and other traditional cardiovascular risk factors, such as blood pressure, lipids and smoking status to estimate 10-year risk of CVD morbidity and mortality, but do not take into account chronic, low-grade inflammation as an independent predictor of CVD. This might in fact account for the gross underestimations of CVD risk when calculators developed for the general population (including SCORE and Framingham Risk Score) are applied in patients with RA, ${ }^{35-37}$ SLE $^{38}$ and PsA. ${ }^{39}$ Notably, systematic differences exist between the general population and populations with IMIDs, who are predominantly female with restricted age ranges.

\section{The Role of Specifically Adapted CVD Risk Calculators}

Especially RA is taken into account as a separate CVD risk factor designated a weight of 1.4 in the QRISK-2 calculator, ${ }^{40,41}$ which was developed in 2008 by adaption of the first QRISK calculator using data from the QRESEARCH database and further updated to QRISK3 in $2017 .^{42}$ Furthermore, the Reynolds Risk Score has been proposed as a more suitable CVD risk calculator in patients with IMIDs as it includes high-sensitivity C-reactive protein (CRP) in the equation among its covariates. $^{43,44}$ The concept of building RA-specific CVD risk calculators to assess patients-tailored CVD risk prediction is not novel. In 2015, the Expanded Risk Score in RA (ERS-RA) was introduced, which was developed using data from a cohort of 23,605 RA patients from the Consortium of Rheumatology Researchers of North America Registry. ${ }^{45}$ ERS-RA combines both traditional CVD risk factors and RA-specific characteristics, such as disease activity, disability and duration, and daily prednisone use. In 2012, A Trans-Atlantic Cardiovascular Consortium for Rheumatoid Arthritis (ATACC-RA) was formed and resulted in the development of two RAspecific risk CVD risk prediction models, using data from 13 cohorts including 5638 RA patients without prior CVD originating from 10 different countries across the world. ${ }^{35}$

Again, not all expectations for improved performance of RA-specific calculators were met. The calculators developed by ATACC-RA did not perform better compared to relevant calculators used in the general population (FRS and ACC/AHA Pooled Cohort Equation in predicting CVD risk among patients with RA). ${ }^{35}$ Using hard CVD endpoints in a cohort of 1796 RA patients without prior CVD, neither QRISK2 nor ERS-RA algorithm was proven more efficient in terms of CVD risk prediction over a mean follow-up of 6.9 years compared to those developed for the general population (ACC/AHA, Framingham Risk Score and Reynolds Risk Score).$^{36}$ In the absence of high performance RA-specific algorithms, it could be assumed that calculators developed for the general population might represent a viable alternative. However, CVD risk was substantially underestimated using either the Reynolds or the Framingham risk score in a populationbased inception cohort of patients with RA of both sexes, especially in older ages and in patients with positive rheumatoid factor. ${ }^{46}$ Altogether, results from application of divergent algorithms in real-world RA cohorts appear rather disappointing considering that neither RA-specific equations nor traditional calculators developed for the general population show sufficient diagnostic accuracy for the early identification of high risk individuals with RA. Failure of RA-specific algorithms to perform better than traditional CVD risk calculators may be largely explained by challenges encountered during their formation and validation. ${ }^{35}$ For example population of interest, ethnicity, and referral bias, as well as study design (longitudinal cohort studies and registries versus randomized clinical studies) and selection of risk factors (traditional versus nontraditional CVD risk factors) have been listed as potential factors affecting, the development of a highly performing CVD risk calculator that would eventually result in improved health outcomes. ${ }^{35}$ Furthermore, incorporation of a single baseline measurement of inflammatory markers such as CRP does not adequately reflect the cumulative burden of chronic, inflammation and does not take into account past and current drug interactions. Subsequently, such modifications did not result in improved CVD risk classification when added to 
Framingham Risk Score equation and the QRISK2 model. ${ }^{47}$

Although efforts to develop specific CVD calculators have almost explicitly focused on RA, where patterns of inflammation-driven atherogenicity have been firmly established, three "SLE-adapted" (modified-SCORE, modified-Framingham Risk Score, QRESEARCH risk estimator, QRISK3) CVD risk scores have been tested in SLE patients in comparison with five generic calculators. However, all of them resulted in underestimation of high CVD risk defined by atherosclerotic plaque presence in patients with SLE. ${ }^{38}$ Likewise, adaption of five CVD risk algorithms according to European League Against Rheumatism (EULAR) indications (SCORE, Framingham Risk Score, QRISK2, Reynold's Risk Score, and the Italian Progetto CUORE individual score) did not result in improved discriminative ability and calibration in patients with PsA. ${ }^{48}$

\section{Relevant Recommendations}

In the light of the above, clinicians are prompted by current EULAR recommendations to perform CVD risk assessment for patients with IMIDs and inflammatory arthropathies according to national guidelines or the SCORE calculator, in case no national guideline is available. However, CVD risk should be further adapted specifically for patients with RA by a 1.5 multiplication factor. Since no conclusive evidence exists regarding the precise CVD risk in patients with AS and PsA, multiplication factor for these diseases is not advocated. CVD risk assessment should be performed at least once every 5 years for all patients with RA, AS or PsA and reassessed following major changes in antirheumatic therapy. ${ }^{17}$ In the same line, current guidelines in the cardiovascular field acknowledge IMIDs as risk modifiers increasing CVD risk estimated by the SCORE system. ${ }^{49}$ The latest 2016 European Guidelines on CVD prevention in clinical practice recommend the use of a 1.5 factor risk multiplier for CVD risk in RA, particularly if disease activity is high, but it may also be considered in other IMIDs on a patient-bypatient basis depending on disease activity and severity. ${ }^{50}$ Still, effective approaches for early identification of high risk individuals are eagerly warranted for patients with IMIDs. Such efforts may not be limited to the development of IMIDs-specific risk prediction models but may incorporate biomarkers or imaging techniques, as will be analyzed in the following.

\section{Application of New Technologies}

Exploitation of novel imaging techniques could potentially facilitate decision-making for CVD monitoring and treatment in patients with IMIDs. Coronary computed tomography angiography (CCTA) has emerged as a non-invasive alternative to selective coronary angiography, which remains the gold standard method for the assessment of coronary artery disease. A recent metaanalysis of 788 patients with RA and 1641 controls provided evidence of more prevalent asymptomatic coronary artery disease, with higher mean coronary calcium score (CCS) derived from CCTA, more multivessel disease, and more high-risk plaques compared to controls. Notably, higher CCS was associated with both RA duration and disease activity. ${ }^{51}$ However, concerns regarding costs and radiation exposure limit generalizability of this method, and it has not been included in the most recent EULAR recommendations for CVD management on patients with RA and other IMIDs. ${ }^{17}$

On the other hand, carotid ultrasound is devoid of these limitations and may help reveal asymptomatic atherosclerosis in patients with RA. More specifically, two features of carotid ultrasound have been traditionally evaluated: presence of atherosclerotic plaques, which has been acknowledged as a coronary heart disease equivalent, ${ }^{52}$ and increased carotid intima-media thickness (cIMT), ie, cIMT $>0.9 \mathrm{~mm}$, which strongly correlates with the degree of atherosclerosis in other vascular beds. ${ }^{53}$ Previous studies have suggested that the addition of carotid ultrasonography may assist CVD risk reclassification of patients with RA and psoriasis into higher CVD risk categories. ${ }^{54-56}$ Therefore, screening for asymptomatic atherosclerotic plaques with carotid ultrasound may be considered as part of the CVD risk evaluation in patients with RA, according to EULAR recommendations. ${ }^{17}$

However, concerns regarding the potential role of carotid ultrasound in terms of CVD risk prediction have also been raised. Measurements of cIMT are highly operatordependent, while plain assessment of the presence of carotid plaques does not allow for tissue characterization through the evaluation of morphological variations in atherosclerotic plaque components within blood vessel walls. To this end, novel methods of analyzing images obtained from carotid ultrasonography have been introduced. Artificial intelligence techniques include machinelearning (ML) and deep-learning (DL) based algorithms with promising potential for tailoring predictive medicine 
to the individual patient. Using these approaches, several patient-specific characteristics can be exploited and incorporated into risk prediction models. Quantitative carotid features extracted from B-mode ultrasound imaging include average, minimum and maximum cIMT, variability in cIMT, and morphological total plaque area, but may also adapt information on lumen diameter, stenosis severity index and inter-adventitial diameter. ${ }^{57}$ In contrast with nearly all popular risk prediction models, which are almost explicitly based on the same set of traditional CVD risk factors, artificial intelligence-based ML algorithms further incorporate carotid image phenotype measurements and have the ability to learn jointly from the training patterns of input risk factors, identify complex and non-linear patterns between variables and capture their association with predicted and observed outcomes. ${ }^{58}$ It has been suggested that ML-based predictive models can provide more accurate CVD risk prediction and subsequently assist physicians in making clinical decisions. In a large and highly heterogenous cohort of 378,256 UK primary care patients, Weng et al showed that ML algorithms perform better compared to an established risk prediction approach at predicting the absolute number of CVD cases correctly. ${ }^{59}$ Likewise, Kakadiaris et al, using a 13-year follow up data set from MESA (the Multi-Ethnic Study of
Atherosclerosis), found that the ML Risk Calculator is more accurate than the ACC/AHA Risk Calculator by recommending less drug therapy and missing fewer events at the same time. ${ }^{60}$ More recently, it was shown that MLbased CVD/stroke risk calculator was superior in terms of 10 -year CVD/stroke risk prediction, compared to the 13 different types of statistically derived risk calculators. ${ }^{61}$ Although these data are encouraging, additional studies are warranted to validate artificial intelligence CVD risk prediction models in specific cohorts such as patients with RA and other IMIDs, and evaluate their potential as clinical aid towards more effective, personalized decisionmaking.

\section{CVD Risk Management in IMIDs}

The main domains of CVD risk management in patients with IMIDs are presented in Figure 2. In detail, the management of CVD risk in IMIDs is based on nonpharmacological interventions namely lifestyle modifications, coupled with medical treatment of traditional CVD risk factors, as well as effective control of disease activity with DMARDs. However, the latter may exert divergent effects on the cardiovascular system regardless of their clinical efficacy, which should be further taken into account.

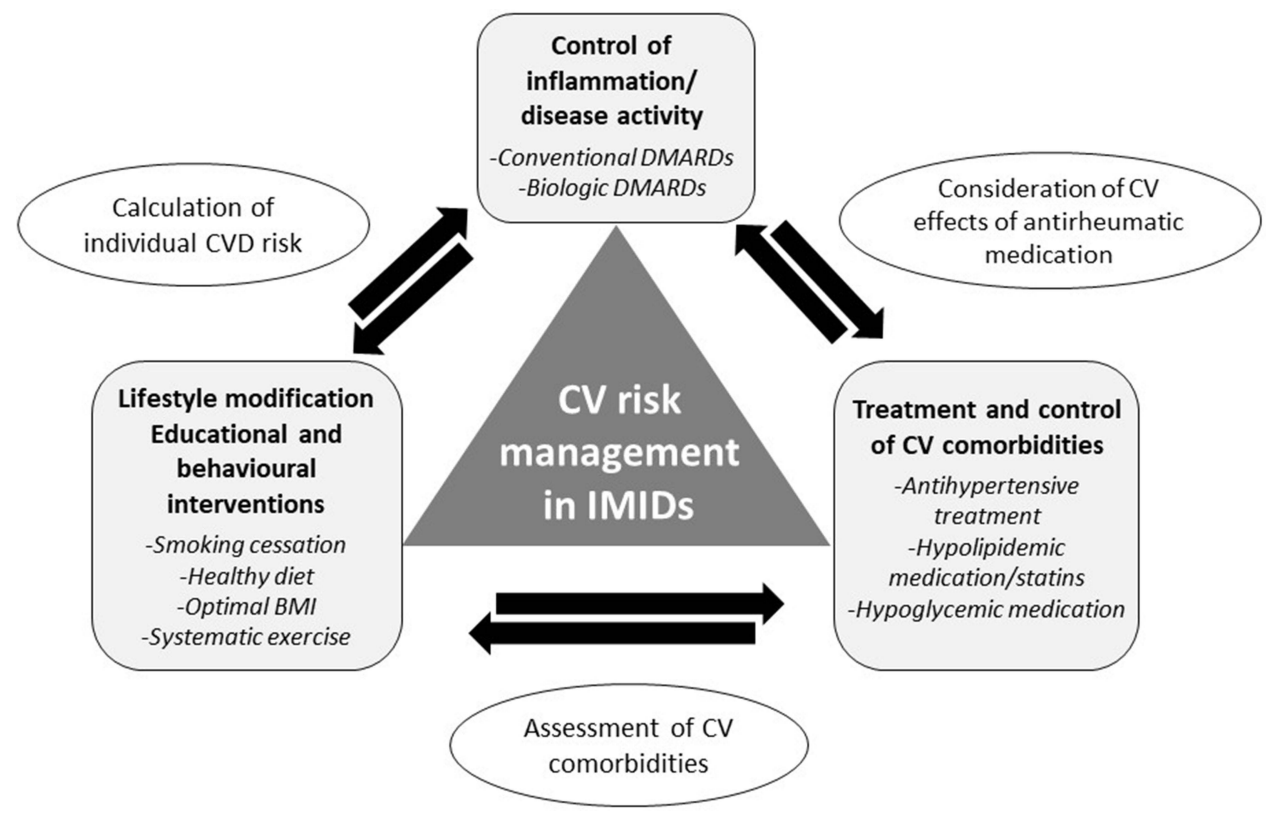

Figure 2 The three pillars of cardiovascular disease (CVD) risk management in patients with IMIDs: I) effective control of inflammation, 2) lifestyle modifications targeting at modifiable CVD risk factors, and 3) simultaneous control of cardiovascular comorbidities. Positive interactions between these interventions are expected not only towards modulation of CVD risk, but also towards substantial improvement of patients' general health and well-being. At the same time, individual CVD risk needs to be determined and regularly reassessed according to the presence of cardiovascular comorbidities, the current and cumulative inflammatory load, and the cardiovascular effects of antirheumatic medication. 


\section{Lifestyle Modification}

A number of modifiable lifestyle factors are potent predictors of future CVD events. Although frequently neglected by health care providers, lifestyle modifications are emerging as a crucial component of IMIDs management regarding both musculoskeletal and CVD outcomes. To this end, educational and behavioural interventions aiming at lifestyle modification are urgently warranted for people with musculoskeletal disability towards primary and secondary CVD prevention. ${ }^{62}$

Smoking is a major risk factor for the development of CVD but may also predispose to altered immunogenetic responses and attenuate the efficacy of anti-rheumatic treatments. ${ }^{63}$ Remarkably, in a multicenter study of 5638 patients with RA and no prior CVD, smoking and hypertension had the highest population attributable risk among traditional CVD risk factors. In the same study, smoking cessation in patients with RA was associated with lower disease activity, improved lipid profiles, and decreased rates of CVD events. ${ }^{64}$ Therefore, patients with IMIDs who smoke should be strongly encouraged to attend smoking cessation programs.

Cardiovascular benefits of a healthy body mass index (BMI) maintenance and a healthy, balanced diet are well established. Patients with IMIDs they are predisposed to changes in body composition, including accumulation of body fat mass and abdominal adiposity. ${ }^{65}$ Therefore, they should monitor their body weight and avoid obesity and overweight through a balanced diet and efforts to lose weight, when necessary. Patients with IMIDs should be advocated to adjust their dietary habits to the principles of the Mediterranean diet, which has been proposed as an ideal nutritional model substantially contributing to the prevention of CVD. ${ }^{66}$ The Mediterranean diet has not only been associated with a less atherogenic profile among patients with RA, but has also been found to alleviate pain and improve inflammatory markers. ${ }^{67,68}$ Although healthy dietary components could complement pharmacological treatment of patients with autoimmune rheumatic disorders, the anti-inflammatory, anti-oxidant effects of single nutritional agents are still being investigated. ${ }^{69}$

Exercise is one of the most significant behavioral interventions that can drastically modulate CVD risk. Unfortunately, patients with IMIDs are prone to sedentary lifestyle and avoid exposure to moderate- to intense exercise. ${ }^{62,70}$ Pain and fatigue have been longitudinally associated with sedentary and standing time in RA. ${ }^{71}$ However, it is biologically plausible that the antiinflammatory effects of exercise may have a beneficial impact not only on CVD risk, but also on rheumatologic outcomes in patients with IMIDs. ${ }^{72}$ Various modes of low to high exercise are beneficial in improving disease-related characteristics and functional status in patients with RA. ${ }^{73}$ Individualized aerobic and resistance exercise intervention may significantly improve cardiorespiratory fitness and individual CVD risk factors, on top of their positive impact on disease activity and severity in RA patients. ${ }^{74}$ Further exploring this study field, Metsios et al confirmed that an exercise program, specifically designed to meet the individual needs and physical abilities of RA patients, improved microvascular and macrovascular endothelial function apart from disease-related characteristics. ${ }^{75,76}$ Additional benefits of systematic exercise include improvement in psychological well-being, ${ }^{77}$ which may be severely affected in patients with IMIDs. ${ }^{78}$ For these reasons, the importance of physical activity has been greatly emphasized by EULAR not only in the 2015/ 2016 updated recommendations for CVD risk management in patients with RA and other forms of inflammatory joint disorders, ${ }^{79}$ but also in the recent 2021 recommendations for the implementation of self-management strategies in patients with inflammatory arthritis, as an effective, feasible and safe tool for managing common comorbidities.

\section{Pharmacological Management}

\section{Antirheumatic Treatment}

Optimal control of inflammation is the cornerstone of the IMIDs management and is clearly associated with restoration of physical function, prevention of irreversible damage in joints and internal organs and improved long term outcomes including survival. Antirheumatic medication cover a broad range of drug classes namely conventional, biologic and synthetic DMARDs, steroids and non-steroid anti-inflammatory drugs (NSAIDs). Despite their clinical efficacy, antirheumatic drugs may exert undesirable effects on the cardiovascular system which should be acknowledged and taken into account while on treatment. ${ }^{20}$ In case of adverse effects that exacerbate CVD risk factors and comorbidities, for instance, antirheumatic agents with blood-pressure raising properties that induce significant elevations in blood pressure or deregulate pre-existing hypertension, the need for administration of such agents should be revisited. Briefly, 
Table I Positive and Negative Effects of Commonly Prescribed Antirheumatic Medication on the CV System

\begin{tabular}{|l|l|}
\hline $\begin{array}{l}\text { NSAIDs (selective/ } \\
\text { non selective) }\end{array}$ & $\begin{array}{l}\text { - Blood pressure elevation-Hypertension } \\
- \text { CV events (heart failure, acute myocardial } \\
\text { infarction, sudden death) }\end{array}$ \\
\hline Glucocorticoids & $\begin{array}{l}\text { - Impaired metabolic profile (hyperglycemia, } \\
\text { insulin resistance)-Prediabetes-Diabetes } \\
- \text { Body fat redistribution-obesity } \\
- \text { Accelerated atherosclerosis } \\
- \text { Blood pressure elevation-Hypertension } \\
- \text { Increased incidence of CV events }\end{array}$ \\
\hline Methotrexate & $\begin{array}{l}- \text { Improvement of metabolic syndrome } \\
\text { components } \\
- \text { Anti-atherogenic effects } \\
- \text { Improved CV outcomes (major CV events, } \\
\text { CV mortality) } \\
- \text { Hyperhomocysteinemia }\end{array}$ \\
\hline Hydroxychloroquine & $\begin{array}{l}\text { - Beneficial effects on metabolic profile and } \\
\text { related comorbidities (diabetes, } \\
\text { dyslipidaemia) } \\
- \text { Decreased rates of CV events }\end{array}$ \\
\hline Biologic DMARDs & $\begin{array}{l}\text { - Blood pressure elevation-Hypertension } \\
- \text { Increased risk of CV and cerebrovascular } \\
\text { events }\end{array}$ \\
\hline Cyclosporine & $\begin{array}{l}\text { events (myocardial infarction, stroke, and } \\
\text { major adverse cardiac events) } \\
- \text { Possible deterioration of hypertension } \\
- \text { Increased lipid levels }\end{array}$ \\
\hline
\end{tabular}

Abbreviations: CV, cardiovascular; DMARDs, disease-modifying antirheumatic drugs; NSAIDs, non-steroidal anti-inflammatory drugs; JAK, janus kinase.

cardiovascular effects of antirheumatic medication are summarized in Table 1.

\section{NSAIDSs}

NSAIDs are classified as non-selective cyclooxygenase (COX) inhibitors (COX-1 and COX-2) or selective (COX-2) inhibitors and are frequently prescribed in patients with IMIDs to alleviate pain and inflammation. Both have been associated with adverse cardiovascular effects, including congestive heart failure ${ }^{80}$ myocardial infarction and even sudden death, with responses varying among different drugs. ${ }^{81,82}$ Moreover, NSAIDs frequently induce elevations in blood pressure and exacerbate pre- existing hypertension. ${ }^{83,84}$ However, CVD risk associated with these drugs may vary. For example, naproxen, a nonselective COX inhibitor, appears least harmful in terms of cardiovascular health compared to other non-selective NSAIDs or coxibs. ${ }^{85}$ The widely advocated belief that naproxen results in better cardiovascular outcomes was nonetheless disputed by the PRECISION trial, which showed that celecoxib, a selective COX-2 inhibitor, was non-inferior with regard to cardiovascular safety, compared to both naproxen and ibuprofen. ${ }^{86}$

\section{Glucocorticoids}

The adverse effects of glucocorticoids on CVD risk factors have been well described. Relatively high daily prednisone doses (starting from $8 \mathrm{mg} /$ day), a high cumulative dose, and a longer exposure to corticosteroids are all predictors of increased CVD risk associated with their use. ${ }^{87-89}$ Cumulative dose of oral glucocorticoids is associated with increased incidence of hypertension in patients with chronic inflammatory disease. ${ }^{90}$ Even at low doses, glucocorticoids induce hyperglycaemia and body fat redistribution in both diabetic and non-diabetic patients. ${ }^{91,92}$ RA patients treated with glucocorticoids present an increase in insulin resistance or glycemia and a greater risk for myocardial infarction and cerebrovascular events. ${ }^{93,94}$ Likewise, high doses of prednisone in patients with SLE have been associated with hyperlipidaemia, hypertension and a greater incidence of coronary artery disease,,${ }^{95,96}$ as well as an increased prevalence of atherosclerotic plaques in the carotid arteries. ${ }^{97}$

\section{Conventional DMARDs}

Methotrexate is the first-line drug for many IMIDs and has been found to potentially decrease mortality, especially CVD-related deaths. Methotrexate was associated with a $21 \%$ lower risk of total CVD and an $18 \%$ lower risk of MI in patients with RA, psoriasis, or polyarthritis, ${ }^{98}$ and might provide a protective effect against atherosclerosis and thrombosis. ${ }^{99}$ Nonetheless, methotrexate decreases the availability of reduced folates, which in turn affects the metabolism of homocysteine. Hyperhomocysteinemia is related to a greater chance of thrombosis and has been proven to significantly increase CVD risk due to the detrimental effect of homocysteine on the vascular endothelium. ${ }^{100,101}$ These adverse effects may be prevented through coadministration of folic acid. ${ }^{102}$ It is worth mentioning that in a well-defined population of very high CVD risk with documented CVD (previous 
myocardial infarction or multivessel coronary disease) who additionally had either type 2 diabetes or the metabolic syndrome, low-dose methotrexate did not result in fewer cardiovascular events compared to placebo. ${ }^{103}$ Thus, it might be assumed that administration of methotrexate cannot reverse established cardiovascular disease, or that the favourable effects of methotrexate on the cardiovascular system are limited to clinical situations in which inflammation levels are high, such as patients with IMIDs. On the other hand, methotrexate-related reduction of CVD risk in patients with RA was not mediated through reductions in disease activity. These findings suggest that CVD risk may be modulated by alternative methotrexaterelated mechanisms in this population. ${ }^{104}$

Hydroxychloroquine is an antimalarial drug, particularly effective in RA and SLE. It is beneficial for the metabolic profile and, to a lesser extent, for reducing the rates of CVD events in patients with RA. ${ }^{105}$ A number of studies have demonstrated the beneficial impact of hydroxychloroquine in diabetes mellitus and dyslipidaemia in patients with autoimmune diseases. ${ }^{106,107}$ These results were again verified in another cohort study of 121,280 patients with RA or psoriasis, treated with hydroxychloroquine or a TNF- $\alpha$ inhibitor, compared to those administered different non-biologic DMARDs. ${ }^{108}$

Finally, cyclosporine is an immunosuppressive agent particularly useful in the treatment of psoriasis and RA, which can induce significant elevations in blood pressure. This increase appears to be dose-related and clinically significant, and amplifies the risk of stroke, myocardial infarction, heart failure and other cardiovascular manifestations attributable to hypertension. ${ }^{109}$ Similarly, hypertension is a common side effect of leflunomide, an isoxazole agent with disease modifying properties commonly prescribed for the treatment of RA. ${ }^{110}$

\section{Biologic DMARDs}

Accumulating evidence suggests a possible reduction in CVD risk mediated by biologic agents, attributable to the improvement of endothelial function, the decrease in inflammation and the reduction of insulin resistance associated with their use. ${ }^{19}$ In particular, treatment with antiTNF- $\alpha$ agents in patients with RA, psoriasis and PsA has been associated in relevant meta-analyses with significant reductions in the risk of all CVD events, including myocardial infarction, stroke, and major adverse cardiac events, ${ }^{111,112}$ although blood-pressure lowering properties have not been consistently reported and relevant information on newest biological treatments is not definite. ${ }^{113,114}$ Furthermore, biologic DMARDs may promote atherogenic lipid profile of patients with RA by increasing the levels of high density lipoprotein cholesterol (HDL-C), low density lipoprotein cholesterol (LDL$\mathrm{C}$ ), and triglycerides. Lipids may have paradoxical associations with the risk of CVD in RA, whereby lower cholesterol and LDL-C levels are associated with increased CVD risk. ${ }^{115}$ This effect is more pronounced following treatment with IL-6 inhibitor tocilizumab but may simply represent a compensatory effect to effective suppression of inflammatory responses without conveying increasing risk for CVD events. ${ }^{116}$

\section{Targeted Synthetic DMARDS}

Janus kinase (JAK) inhibitors are the most recent advanced therapeutic category approved for the management of RA, PsA and ankylosing spondylitis. Despite their remarkable clinical outcomes and the demonstrated superiority over TNF- $\alpha$ inhibitor adalimumab, ${ }^{117}$ a number of concerns have raised regarding their CVD safety profile. $^{118}$ A post marketing surveillance study (NCT02092467) in a selective RA population (age $>50$ year old, with $>1$ CVD risk factor) showed that patients receiving tofacitinib - an unselective JAK1,2,3 inhibitor - had increased risk of serious heart-related events such as heart attack, stroke, or thromboembolic events compared to individuals being treated with TNF- $\alpha$ inhibitors, to the point that a drug safety communication was released by regulators in USA. ${ }^{119}$ By contrast, two recent meta-analyses comprehensively evaluating cardiovascular safety of JAK inhibitors resulted in no significantly elevated CVD risk in patients with either RA or IMIDs, at least in a short-term perspective. ${ }^{120,121}$ Obviously more data are warranted across the whole spectrum of JAK inhibitors before definite conclusions are drawn regarding the profile - if any - of patients at risk for CVD events. However, such observations highlight the complexity and the uncertainty of mechanisms and cytokine pathways implicated in the promotion of vascular injury in IMIDs and further support a comprehensive CVD risk management approach including both diseaseand individuals related characteristics in this population.

\section{Management of Cardiovascular Comorbidities}

In that respect, management of CVD risk factors in patients with IMIDs is of paramount importance. Physicians dealing with rheumatologic patients need to 
be aware of requirements and specific needs of this population. For instance, concomitant antirheumatic medication should be assessed and re-evaluated at an individual basis before deciding on initiation or titration of antihypertensive or lipid lowering regimens, according to the divergent effects of immunosuppressive treatment discussed above. In view of pharmaceutical management of hypertension, dyslipidaemia and diabetes recommendations for the general population apply to patients with IMIDs too.

\section{Hypertension}

According to current European Society of Cardiology/ European Society of Hypertension (ESC/ESH) guidelines, blood pressure targets are currently set to $<130 / 80 \mathrm{mmHg}$ for hypertensive patients $<65$ years and $<140 / 80 \mathrm{mmHg}$ for those $>65$ years. ${ }^{49}$ Current hypertension guidelines do not favor any drug class specifically for patients with IMIDs. However, angiotensin converting enzyme (ACE) inhibitors and angiotensin II receptor blockers (ARBs) appear as a reasonable first-choice treatment for patients with RA who present amplified sympathetic activity, subsequently resulting in high plasma renin activity. ${ }^{122}$ In addition, these drugs appear to downregulate proinflammatory mediators, such as reactive oxygen species and $\mathrm{CRP}$, while at the same time enhance the expression of anti-inflammatory agents. ${ }^{123}$ Beta-blockers and calcium channel blockers are preferred in women of child-bearing potential, ${ }^{49}$ as is often the case in young women with IMIDs. When Raynaud's phenomenon is present, calciumchannel blockers, ACE inhibitors and ARBs are recommended for treatment of concomitant hypertension, while carvedilol and nebivolol should be preferred over selective beta-blockers due to their vasodilatory properties. ${ }^{83}$

\section{Dyslipidaemia}

According to the current European Society of Cardiology/ European Atherosclerosis Society, LDL-C targets are defined according to patients' estimated CVD risk. Specifically, an LDL-C reduction of $\geq 50 \%$ and an LDL$\mathrm{C}$ goal of $<1.4 \mathrm{mmol} / \mathrm{L}(<55 \mathrm{mg} / \mathrm{dl})$ is recommended for secondary prevention in patients with a past CVD event, and for primary prevention for very high CVD risk patients. ${ }^{52}$ For high risk patients, an LDL-C reduction greater than $50 \%$ from baseline and an LDL-C goal $<1.8$ $\mathrm{mmol} / \mathrm{L}(<70 \mathrm{mg} / \mathrm{dl})$ should be achieved. For moderate and low risk patients, an LDL-C goal of $<2.6 \mathrm{mmol} / \mathrm{L}$ $(<100 \mathrm{mg} / \mathrm{dl})$ and $<3 \mathrm{mmol} / \mathrm{L}(<116 \mathrm{mg} / \mathrm{dl})$, respectively, is recommended. Statins remain the cornerstone of pharmacological treatment of dyslipidaemia. ${ }^{52}$ In a randomized, double-blind, placebo-control trial of 3002 RA patients, atorvastatin reduced LDL-C by $0.77 \mathrm{mmol} / \mathrm{L}$ and was associated with significantly lower levels of CRP. ${ }^{124}$ When statins fail to achieve the desired therapeutic targets, a cholesterol absorption inhibitor such as ezetimibe may be added. ${ }^{52}$ Lastly, the proprotein convertase subtilisin/kexin type 9 (PCSK9) human monoclonal antibodies have recently been approved and present pronounced antiatherogenic effects by decreasing LDL-C by as much as $60 \%$ and triglycerides by $26 \%{ }^{125}$ However, antirheumatic treatment frequently includes biological treatments with monoclonal antibodies and future studies are warranted to determine the efficacy and particularly safety of PCSK9 inhibitors in patients with IMIDs.

\section{Diabetes}

Increased incidence of insulin resistance, prediabetes and diabetes is observed in IMIDs, particularly RA, PsA and SLE, ${ }^{126-128}$ and have been linked to chronic inflammation and glucocorticoid use, especially in doses exceeding $7.5 \mathrm{mg}$ daily. ${ }^{129,130}$ However, no large, prospective studies have specifically assessed the efficacy and safety different glucose-lowering agents in IMIDs. General recommendations apply with a HbA1c target of $<7 \%$ to avoid hypoglycemia as well as micro- and macrovascular complications. ${ }^{131}$

\section{Summary: A Practical Approach}

CVD commonly complicates IMIDs and exacerbate disease morbidity and mortality as a result of chronic inflammation and the higher prevalence of traditional CVD risk factors. Optimal disease management requires a careful evaluation of individual CVD risk factors, such as dyslipidaemia, hypertension and impaired glucose tolerance. Regular evaluation of vascular and metabolic abnormalities alongside the tight control of disease activity are warranted. Most importantly, physicians need to develop a holistic approach and assess CVD risk at an individual level. Relevant recommendations apply for the estimation of each patients' CVD risk, but in the lack of accurate calculators specifically designed for patients with IMIDs, application of new technologies could potentially facilitate decision-making for CVD monitoring and treatment. Lifestyle advice and behavioural interventions should be encouraged at any stage of the disease, with emphasis placed on smoking cessation, maintenance of healthy dietary habits and BMI, as well as improvement of cardiorespiratory fitness through introduction systematic exercise. A careful review of 
antirheumatic medication is particularly useful in light of their CVD implications, and changes might be considered in case of pronounced adverse CVD effects. Lastly, physicians should not hesitate to initiate cardiological treatment as indicated. A treat-to-target strategy according to the recommended target goals of dyslipidaemia, hypertension, and diabetes, could confer incremental benefits in the effective management of CVD risk in patients with IMIDs.

\section{Author Contributions}

All authors made substantial contributions to conception and design, acquisition of data, or analysis and interpretation of data; took part in drafting the article or revising it critically for important intellectual content; agreed to submit to the current journal; gave final approval of the version to be published; and agree to be accountable for all aspects of the work. All the contributing authors have read and approved the final edition of the manuscript.

\section{Disclosure}

Panagiota Anyfanti has received funding by Greece and the European Union (European Social Fund- ESF) through the Operational Programme "Human Resources Development, Education and Lifelong Learning 20142020 " in the context of the project. "Evaluation of novel markers of endothelial dysfunction and thrombotic microenvironment in patients with rheumatoid arthritis: association with markers of subclinical inflammation and cardiovascular damage. (MIS 5047870)".

\section{References}

1. Pincus T, Gibson KA, Block JA. Premature mortality: a neglected outcome in rheumatic diseases? Arthritis Care Res. 2015;67:1043-1046. doi:10.1002/acr.22554

2. Lerang K, Gilboe I-M, Steinar Thelle D, Gran JT. Mortality and years of potential life loss in systemic lupus erythematosus: a population-based cohort study. Lupus. 2014;23:1546-1552. doi:10.1177/0961203314551083

3. Avina-Zubieta JA, Thomas J, Sadatsafavi M, Lehman AJ, Lacaille D. Risk of incident cardiovascular events in patients with rheumatoid arthritis: a meta-analysis of observational studies. AnnRheum Dis. 2012;71:1524-1529. doi:10.1136/annrheumdis-2011-200726

4. Bartels CM, Buhr KA, Goldberg JW, et al. Mortality and cardiovascular burden of systemic lupus erythematosus in a US population-based cohort. $J$ Rheumatol. 2014;41:680-687. doi:10.3899/jrheum.130874

5. Jeganathan N, Nguyen E, Sathananthan M. Rheumatoid arthritis and associated-interstitial lung disease: mortality rates and trends. Ann Am Thorac Soc. 2021. doi:10.1513/AnnalsATS.202102-115OC

6. Atienza-Mateo B, Remuzgo-Martínez S, Mora Cuesta VM, et al. The spectrum of interstitial lung disease associated with autoimmune diseases: data of a 3.6-year prospective study from a referral center of interstitial lung disease and lung transplantation. J Clin Med. 2020;9:1606. doi:10.3390/jcm9061606
7. Lee KS, Kronbichler A, Eisenhut M, Lee KH, Shin J. Cardiovascular involvement in systemic rheumatic diseases: an integrated view for the treating physicians. Autoimmun Rev. 2018;17:201-214. doi:10.1016/j.autrev.2017.12.001

8. Nurmohamed MT, Heslinga M, Kitas GD. Cardiovascular comorbidity in rheumatic diseases. Nat Rev Rheumatol. 2015;11:693-704. doi:10.1038/nrrheum.2015.112

9. Psarras A, Soulaidopoulos S, Garyfallos A, Kitas G, Dimitroulas T. A critical view on cardiovascular risk in systemic sclerosis. Rheumatol Int. 2017;37:85-95. doi:10.1007/s00296-016-3530-3

10. Bacons PA, Kitas GD. The significance of vascular inflammation in rheumatoid arthritis. Ann Rheum Dis. 1994;53:621-623. doi:10.1136/ard.53.10.621

11. Semb AG, Rollefstad S, Provan SA, et al. Carotid plaque characteristics and disease activity in rheumatoid arthritis. J Rheumatol. 2013;40:359-368. doi:10.3899/jrheum.120621

12. Panoulas VF, Metsios GS, Pace AV, et al. Hypertension in rheumatoid arthritis. Rheumatology. 2008;47:1286-1298. doi:10.1093/ rheumatology/ken 159

13. Toms E, Symmons D, Kitas G. Dyslipidaemia in rheumatoid arthritis: the role of inflammation, drugs, lifestyle and genetic factors. Curr Vasc Pharmacol. 2010;8:301-326. doi:10.2174/ 157016110791112269

14. Stavropoulos-Kalinoglou A, Metsios GS, Koutedakis Y, Kitas GD; Antonios Stavropoulos-Kalinoglou GSMYKGDKitas. Body-size phenotypes and cardiometabolic risk in rheumatoid arthritis (short title: subgroups of obesity in RA). Mediterr J Rheumatol. 2016;27:48-54. doi:10.31138/mjr.27.2.48

15. O’Brien C, Duda J, Kitas G, van Zanten J, Metsios G, Fenton S. Correlates of sedentary behaviour and light physical activity in people living with rheumatoid arthritis: protocol for a longitudinal study. Mediterr J Mediterr J Rheumatol. 2018;29:106-117. doi:10.31138/mjr.29.2.106

16. Soehnlein O, Libby P, Zimmerman Z, Outterson K. Targeting inflammation in atherosclerosis - from experimental insights to the clinic. Nat Rev Drug Discov. 2021;20:1-22. doi:10.1038/ d41573-020-00185-y

17. Agca R, Heslinga SC, Rollefstad S, et al. EULAR recommendations for cardiovascular disease risk management in patients with rheumatoid arthritis and other forms of inflammatory joint disorders: 2015/2016 update. Ann Rheum Dis. 2017;76:17-28. doi:10.1136/annrheumdis-2016-209775

18. Semb AG, Ikdahl E, Wibetoe G, Crowson C, Rollefstad S. Atherosclerotic cardiovascular disease prevention in rheumatoid arthritis. Nat Rev Rheumatol. 2020;16:361-379. doi:10.1038/ s41584-020-0428-y

19. Fragoulis GE, Soulaidopoulos S, Sfikakis PP, Dimitroulas T, Kitas GD. Effect of biologics on cardiovascular inflammation: mechanistic insights and risk reduction. $J$ Inflamm Res. 2021;14:1915-1931. doi:10.2147/JIR.S282691

20. Yuri Gasparyan A, Ayvazyan L, Cocco G, Kitas D. Adverse cardiovascular effects of antirheumatic drugs: implications for clinical practice and research. Curr Pharm Des. 2012;18:1543-1555. doi:10.2174/138161212799504759

21. Roifman I, Beck PL, Anderson TJ, Eisenberg MJ, Genest J. Chronic inflammatory diseases and cardiovascular risk: a systematic review. Can J Cardiol. 2011;27:174-182. doi:10.1016/j.cjca.2010.12.040

22. Bacon PA, Stevens RJ, Carruthers DM, Young SP, Kitas GD. Accelerated atherogenesis in autoimmune rheumatic diseases. Autoimmun Rev. 2002;1:338-347. doi:10.1016/S1568-9972(02) 00100-3

23. Anyfanti P, Gavriilaki E, Douma S, Gkaliagkousi E. Endothelial dysfunction in patients with rheumatoid arthritis: the role of hypertension. Curr Hypertens Rep. 2020;22. doi:10.1007/ s11906-020-01064-y 
24. Anyfanti P, Gkaliagkousi E, Gavriilaki E, et al. Association of galectin-3 with markers of myocardial function, atherosclerosis, and vascular fibrosis in patients with rheumatoid arthritis. Clin Cardiol. 2019;42:62-68. doi:10.1002/clc.23105

25. Dimitroulas T, Hodson J, Sandoo A, Smith J, Kitas GD. Endothelial injury in rheumatoid arthritis: a crosstalk between dimethylarginines and systemic inflammation. Arthritis Res Ther. 2017;19:32.

26. Sandoo A, Dimitroulas T, Hodson J, Smith JP, Douglas KM, Kitas GD. Cumulative inflammation associates with asymmetric dimethylarginine in rheumatoid arthritis: a 6 year follow-up study. Rheumatology. 2015;54:1145-1152. doi:10.1093/rheumatology/ keu349

27. Anyfanti P, Triantafyllou A, Gkaliagkousi E, et al. Subendocardial viability ratio in patients with rheumatoid arthritis: comparison with healthy controls and identification of prognostic factors. Clin Rheumatol. 2017;36:1229-1236. doi:10.1007/ s10067-017-3659-9

28. Anyfanti P, Triantafyllou A, Gkaliagkousi E, et al. Retinal vessel morphology in rheumatoid arthritis: association with systemic inflammation, subclinical atherosclerosis, and cardiovascular risk. Microcirculation. 2017;24:e12417. doi:10.1111/micc.12417

29. Anyfanti P, Gkaliagkousi E, Triantafyllou A, et al. Dermal capillary rarefaction as a marker of microvascular damage in patients with rheumatoid arthritis: association with inflammation and disorders of the macrocirculation. Microcirculation. 2018;25: e12451. doi:10.1111/micc.12451

30. Arida A, Protogerou A, Kitas G, Sfikakis P. Systemic inflammatory response and atherosclerosis: the paradigm of chronic inflammatory rheumatic diseases. Int J Mol Sci. 2018;19:1890. doi:10.3390/ijms 19071890

31. Kitas GD, Dimitroulas T. Cardiovascular comorbidity in rheumatic and musculoskeletal diseases: where we are and how can we move forward. Int $J$ Rheum Dis. 2021;24:473-476. doi:10.1111/1756-185X.14112

32. Conroy R. Estimation of ten-year risk of fatal cardiovascular disease in Europe: the SCORE project. Eur Heart $J$. 2003;24:987-1003. doi:10.1016/S0195-668X(03)00114-3

33. D'Agostino RB, Vasan RS, Pencina MJ, et al. General cardiovascular risk profile for use in primary care. Circulation. 2008;117:743-753. doi:10.1161/CIRCULATIONAHA.107.699579

34. Goff DC, Lloyd-Jones DM, Bennett G, et al. 2013 ACC/AHA guideline on the assessment of cardiovascular risk. $\mathrm{J} \mathrm{Am} \mathrm{Coll}$ Cardiol. 2014;63:2935-2959. doi:10.1016/j.jacc.2013.11.005

35. Crowson CS, Rollefstad S, Kitas GD, van Riel PL, Gabriel SE, Semb AG. Challenges of developing a cardiovascular risk calculator for patients with rheumatoid arthritis. Arthritis Rheumatol. 2017;67:1995-2003. doi:10.1371/journal.pone.0174656

36. Crowson CS, Gabriel SE, Semb AG, et al. Rheumatoid arthritis-specific cardiovascular risk scores are not superior to general risk scores: a validation analysis of patients from seven countries. Rheumatology. 2017;56:1102-1110. doi:10.1093/rheumatology/kex038

37. Wahlin B, Innala L, Magnusson S, et al. Performance of the expanded cardiovascular risk prediction score for rheumatoid arthritis is not superior to the ACC/AHA risk calculator. J Rheumatol. 2019;46:130-137. doi:10.3899/jrheum.171008

38. Drosos GC, Konstantonis G, Sfikakis PP, Tektonidou MG. Underperformance of clinical risk scores in identifying vascular ultrasound-based high cardiovascular risk in systemic lupus erythematosus. Eur J Prev Cardiol. 2021;28:346-352. doi:10.1093/eurjpc/zwaa256

39. Shen J, Lam SH, Shang Q, et al. Underestimation of risk of carotid subclinical atherosclerosis by cardiovascular risk scores in patients with psoriatic arthritis. $J$ Rheumatol. 2018;45:218-226. doi:10.3899/jrheum.170025
40. Hippisley-Cox J, Coupland C, Vinogradova Y, Robson J, May M, Brindle P. Derivation and validation of QRISK, a new cardiovascular disease risk score for the United Kingdom: prospective open cohort study. BMJ. 2007;335:136. doi:10.1136/ bmj.39261.471806.55

41. Hippisley-Cox J, Coupland C, Vinogradova Y, et al. Predicting cardiovascular risk in England and Wales: prospective derivation and validation of QRISK2. BMJ. 2008;336:1475-1482. doi:10.1136/bmj.39609.449676.25

42. Hippisley-Cox J, Coupland C, Brindle P. Development and validation of QRISK3 risk prediction algorithms to estimate future risk of cardiovascular disease: prospective cohort study. BMJ. 2017;357:j2099.

43. Ridker PM, Buring JE, Rifai N, Cook NR. Development and validation of improved algorithms for the assessment of global cardiovascular risk in women. JAMA. 2007;297:611-619. doi:10.1001/jama.297.6.611

44. Ridker PM, Paynter NP, Rifai N, Gaziano JM, Cook NR C-reactive protein and parental history improve global cardiovascular risk prediction. Circulation. 2008;118:2243-2251. doi:10.1161/CIRCULATIONAHA.108.814251

45. Solomon DH, Greenberg J, Curtis JR, et al. Derivation and internal validation of an expanded cardiovascular risk prediction score for rheumatoid arthritis: a consortium of rheumatology researchers of North America registry study. Arthritis Rheumatol. 2015;67:1995-2003. doi:10.1002/art.39195

46. Crowson CS, Matteson EL, Roger VL, Therneau TM, Gabriel SE. Usefulness of risk scores to estimate the risk of cardiovascular disease in patients with rheumatoid arthritis. Am J Cardiol. 2012;110:420-424. doi:10.1016/j.amjcard.2012.03.044

47. Alemao E, Cawston H, Bourhis F, et al. Comparison of cardiovascular risk algorithms in patients with vs without rheumatoid arthritis and the role of C-reactive protein in predicting cardiovascular outcomes in rheumatoid arthritis. Rheumatology. 2017;56:777-786. doi:10.1093/rheumatology/kew440

48. Navarini L, Margiotta DPE, Caso F, et al. Performances of five risk algorithms in predicting cardiovascular events in patients with Psoriatic Arthritis: an Italian bicentric study. Ann Rheum Dis. 2018;77:1034. doi:10.1371/journal.pone.0205506

49. Williams B, Mancia G, Spiering W, et al. 2018 ESC/ESH guidelines for the management of arterial hypertension. $J$ Hypertens. 2018;36:1953-2041. doi:10.1097/HJH.0000000000001940

50. Piepoli MF, Hoes AW, Agewall S, et al. 2016 European guidelines on cardiovascular disease prevention in clinical practice. Eur Heart J. 2016;37:2315-2381. doi:10.1093/eurheartj/ehw106

51. Hansen PR, Feineis M, Abdulla J. Rheumatoid arthritis patients have higher prevalence and burden of asymptomatic coronary artery disease assessed by coronary computed tomography: a systematic literature review and meta-analysis. Eur J Intern Med. 2019;62:72-79. doi:10.1016/j.ejim.2019.02.018

52. Mach F, Baigent C, Catapano AL, et al. 2019 ESC/EAS guidelines for the management of dyslipidaemias: lipid modification to reduce cardiovascular risk. Eur Heart J. 2020;41:111-188. doi:10.1093/eurheartj/ehz455

53. O’Leary DH, Polak JF, Kronmal RA, Manolio TA, Burke GL, Wolfson SK. Carotid-artery intima and media thickness as a risk factor for myocardial infarction and stroke in older adults. $N$ Engl J Med. 1999;340:14-22. doi:10.1056/NEJM199901073400103

54. Dessein PH, Joffe BI, Veller MG, et al. Traditional and nontraditional cardiovascular risk factors are associated with atherosclerosis in rheumatoid arthritis. $J$ Rheumatol. 2005;32:435-442.

55. Corrales A, González-Juanatey C, Peiró ME, Blanco R, Llorca J, González-Gay MA. Carotid ultrasound is useful for the cardiovascular risk stratification of patients with rheumatoid arthritis: results of a population-based study. Ann Rheum Dis. 2014;73:722-727. doi:10.1136/annrheumdis-2012-203101 
56. Eder L, Chandran V, Gladman DD. The Framingham Risk Score underestimates the extent of subclinical atherosclerosis in patients with psoriatic disease. Ann Rheum Dis. 2014;73:1990-1996. doi:10.1136/annrheumdis-2013-203433

57. Jamthikar A, Gupta D, Khanna NN, et al. A special report on changing trends in preventive stroke/cardiovascular risk assessment via B-mode ultrasonography. Curr Atheroscler Rep. 2019;21:25. doi:10.1007/s11883-019-0788-4

58. Jamthikar AD, Gupta D, Saba L, et al. Artificial intelligence framework for predictive cardiovascular and stroke risk assessment models: a narrative review of integrated approaches using carotid ultrasound. Comput Biol Med. 2020;126:104043. doi:10.1016/j.compbiomed.2020.104043

59. Weng SF, Reps J, Kai J, Garibaldi JM, Qureshi N. Can machine-learning improve cardiovascular risk prediction using routine clinical data? PLoS One. 2017;12:e0174944. doi:10.1371/journal.pone.0174944

60. Kakadiaris IA, Vrigkas M, Yen AA, Kuznetsova T, Budoff M, Naghavi M. Machine learning outperforms ACC/AHA CVD risk calculator in Mesa. J Am Heart Assoc. 2018;20:e009476. doi:10.1161/JAHA.118.009476

61. Jamthikar A, Gupta D, Saba L, et al. Cardiovascular/stroke risk predictive calculators: a comparison between statistical and machine learning models. Cardiovasc Diagn Ther. 2020;10:919-938. doi:10.21037/cdt.2020.01.07

62. Fenton SAM, Duda JL, Veldhuijzen van Zanten JJ, Metsios GS, Kitas GD. Theory-informed interventions to promote physical activity and reduce sedentary behaviour in rheumatoid arthritis: a critical review of the literature. Mediterr $J$ Rheumatol. 2020;31:19-41. doi:10.31138/mjr.31.1.19

63. Sugiyama D, Nishimura K, Tamaki K, et al. Impact of smoking as a risk factor for developing rheumatoid arthritis: a meta-analysis of observational studies. Ann Rheum Dis. 2010;69:70-81. doi:10.1136/ard.2008.096487

64. Crowson CS, Rollefstad S, Ikdahl E, et al. Impact of risk factors associated with cardiovascular outcomes in patients with rheumatoid arthritis. Ann Rheum Dis. 2018;77:48-54. doi:10.1136/ annrheumdis-2017-211735

65. Santos MJ, Vinagre F, Canas da Silva J, Gil V, Fonseca JE. Body composition phenotypes in systemic lupus erythematosus and rheumatoid arthritis: a comparative study of Caucasian female patients. Clin Exp Rheumatol. 2011;29:470-476.

66. Markaki AG, Gkiouras K, Papakitsos C, et al. Disease activity, functional ability and nutritional status in patients with rheumatoid arthritis: an observational study in Greece. Mediterr J Rheumatol. 2020;31:406-411. doi:10.31138/ mjr.31.4.406

67. Hulander E, Bärebring L, Turesson Wadell A, et al. Diet intervention improves cardiovascular profile in patients with rheumatoid arthritis: results from the randomized controlled cross-over trial ADIRA. Nutr J. 2021;20:9. doi:10.1186/s12937-021-00663-y

68. Skoldstam L. An experimental study of a Mediterranean diet intervention for patients with rheumatoid arthritis. Ann Rheum Dis. 2003;62:208-214. doi:10.1136/ard.62.3.208

69. Dahan S, Segal Y, Shoenfeld Y. Dietary factors in rheumatic autoimmune diseases: a recipe for therapy? Nat Rev Rheumatol. 2017;13:348-358. doi:10.1038/nrrheum.2017.42

70. Fenton SAM, Veldhuijzen van Zanten JJ, Duda JL, Metsios GS, Kitas GD. Sedentary behaviour in rheumatoid arthritis: definition, measurement and implications for health. Rheumatology. 2018;57:213-226. doi:10.1093/rheumatology/kex053

71. O’Brien CM, Ntoumanis N, Duda JL, et al. Pain and fatigue are longitudinally and bi-directionally associated with more sedentary time and less standing time in rheumatoid arthritis. Rheumatology. 2021;25:keab029. doi:10.1093/rheumatology/keab029
72. Metsios GS, Moe RH, Kitas GD. Exercise and inflammation. Best Pract Res Clin Rheumatol. 2020;34:101504. doi:10.1016/j. berh.2020.101504

73. Metsios GS, Stavropoulos-Kalinoglou A, Veldhuijzen van Zanten JJ, et al. Rheumatoid arthritis, cardiovascular disease and physical exercise: a systematic review. Rheumatology. 2008;47:239-248. doi:10.1093/rheumatology/kem260

74. Stavropoulos-Kalinoglou A, Metsios GS, Veldhuijzen van Zanten JJ, Nightingale P, Kitas GD, Koutedakis Y. Individualised aerobic and resistance exercise training improves cardiorespiratory fitness and reduces cardiovascular risk in patients with rheumatoid arthritis. Ann Rheum Dis. 2013;72:1819-1825. doi:10.1136/annrheumdis-2012-202075

75. Metsios GS, Moe RH, van der Esch M, et al. The effects of exercise on cardiovascular disease risk factors and cardiovascular physiology in rheumatoid arthritis. Rheumatol Int. 2020;4:347-357. doi:10.1007/s00296-019-04483-6

76. Metsios GS, Stavropoulos-Kalinoglou A, Veldhuijzen van Zanten JJ, et al. Individualised exercise improves endothelial function in patients with rheumatoid arthritis. Ann Rheum Dis. 2014;73:748-751. doi:10.1136/annrheumdis-2013-203291

77. Metsios GS, Stavropoulos-Kalinoglou A, Kitas GD. The role of exercise in the management of rheumatoid arthritis. Expert Rev Clin Immunol. 2015;11:1121-1130. doi:10.1586/1744666X.2015.1067606

78. Anyfanti P, Gavriilaki E, Pyrpasopoulou A, et al. Depression, anxiety, and quality of life in a large cohort of patients with rheumatic diseases: common, yet undertreated. Clin Rheumatol. 2016;35:733-739. doi:10.1007/s10067-014-2677-0

79. Nikiphorou E, Santos EJF, Marques A, et al. 2021 EULAR recommendations for the implementation of self-management strategies in patients with inflammatory arthritis. Ann Rheum Dis. 2021;80:1278-1285. doi:10.1136/annrheumdis-2021-220249

80. Tobias D. Risk of admission for CHF is higher for rofecoxib and nonselective NSAID users. Commentary: cyclo-oxygenase-2 inhibitors versus nonselective, nonsteroidal anti-inflammatory drugs and congestive heart failure outcomes in elderly patients: a population-based cohort study. Consult Pharm. 2004;19:1038-1042. doi:10.4140/tcp.n.2004.1038

81. Ray WA, Stein CM, Daugherty JR, Hall K, Arbogast PG, Griffin MR. COX-2 selective non-steroidal anti-inflammatory drugs and risk of serious coronary heart disease. Lancet (London, England). 2002;360:1071-1073. doi:10.1016/S0140-6736(02)11131-7

82. Hermann M, Ruschitzka F. Coxibs, non-steroidal anti-inflammatory drugs and cardiovascular risk. Intern Med J. 2006;36:308-319. doi:10.1111/j.1445-5994.2006.01056.x

83. Panoulas VF, Metsios GS, Pace AV, et al. Hypertension in rheumatoid arthritis. Rheumatology (Oxford, England). 2008;47:458. doi:10.1093/rheumatology/ken159

84. Whelton A. Renal and related cardiovascular effects of conventional and COX-2-specific NSAIDs and non-NSAID analgesics. $\mathrm{Am}$ J Ther. 2000;7:63-74. doi:10.1097/00045391-200007020-00004

85. Trelle S, Reichenbach S, Wandel S, et al. Cardiovascular safety of non-steroidal anti-inflammatory drugs: network meta-analysis. BMJ. 2011;342:c7086-c7086. doi:10.1136/bmj.c7086

86. Nissen SE, Yeomans ND, Solomon DH, et al. Cardiovascular safety of celecoxib, naproxen, or ibuprofen for arthritis. $N$ Engl J Med. 2016;375(26):2519-2529. doi:10.1056/NEJMoa1611593

87. Del Rincón I, Battafarano DF, Restrepo JF, Erikson JM, Escalante A. Glucocorticoid dose thresholds associated with all-cause and cardiovascular mortality in rheumatoid arthritis. Arthritis Rheumatol. 2014;66:264-272. doi:10.1002/art.38210

88. Ocon AJ, Reed G, Pappas DA, Curtis JR, Kremer JM. Short-term dose and duration-dependent glucocorticoid risk for cardiovascular events in glucocorticoid-naive patients with rheumatoid arthritis. Ann Rheum Dis. 2021;80(12):1522-1529. doi:10.1136/ annrheumdis-2021-220577 
89. Roubille C, Coffy A, Rincheval N, et al. Ten-year analysis of the risk of severe outcomes related to low-dose glucocorticoids in early rheumatoid arthritis. Rheumatology (Oxford, England). 2021;60(8):3738-3746. doi:10.1093/rheumatology/keaa850

90. Mebrahtu TF, Morgan AW, West RM, Stewart PM, PujadesRodriguez M. Oral glucocorticoids and incidence of hypertension in people with chronic inflammatory diseases: a population-based cohort study. CMAJ. 2020;192:295-301. doi:10.1503/cmaj.191012

91. Clore JN, Thurby-Hay L. Glucocorticoid-induced hyperglycemia. Endocr Pract. 2009;15:469-474. doi:10.4158/EP08331.RAR

92. Gurwitz JH, Bohn RL, Glynn RJ, Monane M, Mogun H, Avorn J. Glucocorticoids and the risk for initiation of hypoglycemic therapy. Arch Intern Med. 1994;154:97-101. doi:10.1001/ archinte.1994.00420010131015

93. Ruyssen-Witrand A, Fautrel B, Saraux A, le Loët X, Pham T. Cardiovascular risk induced by low-dose corticosteroids in rheumatoid arthritis: a systematic literature review. Joint Bone Spine. 2011;78:23-30. doi:10.1016/j.jbspin.2010.02.040

94. Xie W, Yang X, Ji L, Zhang Z. Incident diabetes associated with hydroxychloroquine, methotrexate, biologics and glucocorticoids in rheumatoid arthritis: a systematic review and meta-analysis. Semin Arthritis Rheum. 2020;50(4):598-607. doi:10.1016/j. semarthrit.2020.04.005

95. Manzi S, Meilahn EN, Rairie JE, et al. Age-specific incidence rates of myocardial infarction and angina in women with systemic lupus erythematosus: comparison with the Framingham Study. Am J Epidemiol. 1997;145:408-415. doi:10.1093/oxfordjournals. aje.a009122

96. Petri M, Perez-Gutthann S, Spence D, Hochberg MC. Risk factors for coronary artery disease in patients with systemic lupus erythematosus. Am J Med. 1992;93:513-519. doi:10.1016/00029343(92)90578-Y

97. Doria A, Shoenfeld Y, Wu R, et al. Risk factors for subclinical atherosclerosis in a prospective cohort of patients with systemic lupus erythematosus. Ann Rheum Dis. 2003;62:1071-1077. doi:10.1136/ard.62.11.1071

98. Micha R, Imamura F, Wyler von Ballmoos M, et al. Systematic review and meta-analysis of methotrexate use and risk of cardiovascular disease. Am J Cardiol. 2011;108:1362-1370. doi:10. 1016/j.amjcard.2011.06.054

99. Mangoni AA, Zinellu A, Sotgia S, Carru C, Erre GL. Methotrexate and cardiovascular protection: current evidence and future directions. Clin Med Insights Therap. 2017;9:11 79559X17741289.

100. Chan ESL, Cronstein BN. Methotrexate-how does it really work? Nat Rev Rheumatol. 2010;6:175-178. doi:10.1038/nrrheum.2010.5

101. Ganguly P, Alam SF. Role of homocysteine in the development of cardiovascular disease. Nutr J. 2015;14:1. doi:10.1186/14752891-14-6

102. van Ede AE, Laan RF, Blom HJ, et al. Homocysteine and folate status in methotrexate-treated patients with rheumatoid arthritis. Rheumatology. 2002;41:658-665. doi:10.1093/rheumatology/ 41.6.658

103. Ridker PM, Everett BM, Pradhan A, MacFadyen JG, Solomon DH, Zaharris E. Low-dose methotrexate for the prevention of atherosclerotic events. $N$ Engl J Med. 2019;380 (8):752-762. doi:10.1056/NEJMoa1809798

104. Johnson TM, Sayles HR, Baker JF, George MD, Roul P, Zheng C. Investigating changes in disease activity as a mediator of cardiovascular risk reduction with methotrexate use in rheumatoid arthritis. Ann Rheum Dis. 2021;80(11):1385-1392. doi:10.1136/ annrheumdis-2021-220125

105. Rempenault C, Combe B, Barnetche T, et al. Metabolic and cardiovascular benefits of hydroxychloroquine in patients with rheumatoid arthritis: a systematic review and meta-analysis. Ann Rheum Dis. 2018;77:98-103. doi:10.1136/annrheumdis-2017-211836
106. Bili A, Sartorius JA, Kirchner HL, et al. Hydroxychloroquine use and decreased risk of diabetes in rheumatoid arthritis patients. $J$ Clin Rheumatol. 2011;17:115-120. doi:10.1097/RHU.0b013e318214b6b5

107. Hage MP, Al-Badri MR, Azar ST. A favorable effect of hydroxychloroquine on glucose and lipid metabolism beyond its anti-inflammatory role. Ther Adv Endocrinol Metab. 2014;5:77-85. doi:10.1177/2042018814547204

108. Solomon DH. Association between disease-modifying antirheumatic drugs and diabetes risk in patients with rheumatoid arthritis and psoriasis. JAMA. 2011;305:2525-2531. doi:10.1001/jama.2011.878

109. Robert N, Wong GW, Wright JM. Effect of cyclosporine on blood pressure. Cochrane Database Syst Rev. 2010;1:CD007893. doi:10.1002/14651858.CD007893.pub2

110. Rozman B. Clinical experience with leflunomide in rheumatoid arthritis. Leflunomide Investigators' Group. $J$ Rheumatol. 1998;53:27-32.

111. Roubille C, Richer V, Starnino T, et al. The effects of tumour necrosis factor inhibitors, methotrexate, non-steroidal anti-inflammatory drugs and corticosteroids on cardiovascular events in rheumatoid arthritis, psoriasis and psoriatic arthritis: a systematic review and meta-analysis. Ann Rheum Dis. 2015;74:480-489. doi:10.1136/annrheumdis-2014-206624

112. Barnabe C, Martin B-J, Ghali WA. Systematic review and metaanalysis: anti-tumor necrosis factor $\alpha$ therapy and cardiovascular events in rheumatoid arthritis. Arthritis Care Res. 2011;63:522-529. doi:10.1002/acr.20371

113. Sandoo A, Panoulas VF, Toms TE, et al. Anti-TNF $\alpha$ therapy may lead to blood pressure reductions through improved endotheliumdependent microvascular function in patients with rheumatoid arthritis. J Hum Hypertens. 2011;25:699-702. doi:10.1038/jhh.2011.36

114. Zhao Q, Hong D, Zhang Y, Sang Y, Yang Z, Zhang X. Association between anti-TNF therapy for rheumatoid arthritis and hypertension. Medicine. 2015;94:e731. doi:10.1097/ MD.0000000000000731

115. Toms E, Symmons P, Kitas G. Dyslipidaemia in rheumatoid arthritis: the role of inflammation, drugs, lifestyle and genetic factors. Curr Vasc Pharmacol. 2010;8:301-326. doi:10.2174/ 157016110791112269

116. McInnes IB, Thompson L, Giles JT, et al. Effect of interleukin-6 receptor blockade on surrogates of vascular risk in rheumatoid arthritis: MEASURE, a randomised, placebo-controlled study. Ann Rheum Dis. 2015;74:694-702. doi:10.1136/annrheumdis-2013-204345

117. Chatzidionysiou K. Beyond methotrexate and biologics in RA efficacy of JAK inhibitors and their place in the current treatment armamentarium. Mediterr $J$ Rheumatol. 2020;31:120-128. doi:10.31138/mjr.31.1.120

118. Taylor PC, Weinblatt ME, Burmester GR, et al. Cardiovascular safety during treatment with baricitinib in rheumatoid arthritis. Arthritis Rheumatol. 2019;71:1042-1055. doi:10.1002/art.40841

119. FDA requires warnings about increased risk of serious heart-related events, cancer, blood clots, and death for JAK inhibitors that treat certain chronic inflammatory conditions. Available from: https:// www.fda.gov/drugs/drug-safety-and-availability/fda-requireswarnings-about-increased-risk-serious-heart-related-events-cancerblood-clots-and-death. Accessed November 23, 2021.

120. Xie W, Xiao S, Huang Y, Sun X, Zhang Z. Effect of tofacitinib on cardiovascular events and all-cause mortality in patients with immune-mediated inflammatory diseases: a systematic review and meta-analysis of randomized controlled trials. Ther $A d v$ Musculoskelet Dis. 2019;11:1759720X19895492. doi:10.1177/ 1759720X19895492

121. Xie W, Huang Y, Xiao S, Sun X, Fan Y, Zhang Z. Impact of Janus kinase inhibitors on risk of cardiovascular events in patients with rheumatoid arthritis: systematic review and meta-analysis of randomised controlled trials. Ann Rheum Dis. 2019;78 (8):1048-1054. doi:10.1136/annrheumdis-2018-214846 
122. Moreira FRC, de Oliveira TA, Ramos NE, Abreu MAD, Simões E, Silva AC. The role of renin angiotensin system in the pathophysiology of rheumatoid arthritis. Mol Biol Rep. 2021;48:6619-6629. doi:10.1007/s11033-021-06672-8

123. Dandona P, Dhindsa S, Ghanim H and Chaudhuri A. Angiotensin II and inflammation: the effect of angiotensin-converting enzyme inhibition and angiotensin II receptor blockade. Journal of human hypertension. 21, 2007. doi:10.1038/sj.jhh.1002101.

124. Kitas GD, Nightingale P, Armitage J, et al. A multicenter, randomized, placebo-controlled trial of atorvastatin for the primary prevention of cardiovascular events in patients with rheumatoid arthritis. Arthritis Rheumatol. 2019;71:1437-1449. doi:10.1002/art.40892

125. Panoulas V, Kitas GD. Pharmacological management of cardiovascular risk in chronic inflammatory rheumatic diseases. Expert Rev Clin Pharmacol. 2020;13:605-613. doi:10.1080/17512433.2020.1766964

126. Solomon DH, Love TJ, Canning C, Schneeweiss S. Risk of diabetes among patients with rheumatoid arthritis, psoriatic arthritis and psoriasis. Ann Rheum Dis. 2010;69:2114-2117. doi:10.1136/ard.2009.125476

127. Charlton R, Green A, Shaddick G, et al. Risk of type 2 diabetes and cardiovascular disease in an incident cohort of people with psoriatic arthritis: a population-based cohort study. Rheumatology. 2019;58:144-148. doi:10.1093/rheumatology/key286
128. Bruce IN, Urowitz MB, Gladman DD, Ibañez D, Steiner G. Risk factors for coronary heart disease in women with systemic lupus erythematosus: the Toronto Risk Factor Study. Arthritis Rheum. 2003;48:3159-3167. doi:10.1002/art.11296

129. Chung CP, Oeser A, Solus JF, et al. Inflammation-associated insulin resistance: differential effects in rheumatoid arthritis and systemic lupus erythematosus define potential mechanisms. Arthritis Rheum. 2008;58:2105-2112. doi:10.1002/ art. 23600

130. Lillegraven S, Greenberg JD, Reed GW, et al. Immunosuppressive treatment and the risk of diabetes in rheumatoid arthritis. PLoS One. 2019;14:e0210459. doi:10.1371/journal. pone. 0210459

131. Cosentino F, Grant PJ, Aboyans V, et al. 2019 ESC Guidelines on diabetes, pre-diabetes, and cardiovascular diseases developed in collaboration with the EASD. Eur Heart J. 2020;41:4317. doi:10.1093/eurheartj/ehz486
Journal of Inflammation Research

\section{Publish your work in this journal}

The Journal of Inflammation Research is an international, peerreviewed open-access journal that welcomes laboratory and clinical findings on the molecular basis, cell biology and pharmacology of inflammation including original research, reviews, symposium reports, hypothesis formation and commentaries on: acute/chronic inflammation; mediators of inflammation; cellular processes; molecular
Dovepress

mechanisms; pharmacology and novel anti-inflammatory drugs; clinical conditions involving inflammation. The manuscript management system is completely online and includes a very quick and fair peerreview system. Visit http://www.dovepress.com/testimonials.php to read real quotes from published authors. 